\title{
Genome-Wide Linkage Scan Maps ETINPH Gene to Chromosome 19q12-13.31
}

\author{
Jun Zhang ${ }^{\mathrm{a}}$ Christopher W. Carr ${ }^{\mathrm{b}}$ Daniele Rigamontic Ahmed Badr ${ }^{\mathrm{a}}$ \\ a Departments of Anesthesiology and Neurosurgery, Center for Neurosciences, Texas Tech University Health \\ Science Center, El Paso, Tex., ${ }^{b}$ University of Mississippi Medical Center, Jackson, Miss., and \\ 'Department of Neurosurgery, The Johns Hopkins University School of Medicine, Baltimore, Md., USA
}

\section{Key Words}

Essential tremor-idiopathic normal pressure hydrocephalus . ETINPH gene $\cdot$ Linkage analysis

\begin{abstract}
Objective: Essential tremor (ET), one of the most common neurological disorders, comprises uncontrollable tremor, usually in the upper extremities. Idiopathic normal pressure hydrocephalus (iNPH) features ventricular enlargement in the absence of elevated intracranial pressure; its three cardinal symptoms are gait impairment, incontinence, and dementia. iNPH is among the most common medical problems in the older population. To date, the genetic etiologies of ET and iNPH remain largely elusive. Methods: We previously identified a large kindred in which the affected members developed ET in adolescence or young adulthood and $\mathrm{NPH}$ when elderly, in that case called ETINPH. Employing two different genotyping microarrays and two- or multipoint linkage analysis strategies, genome-wide linkage scans were performed. Results: We mapped the ETINPH locus to chromosome 19q12-13.31. Fine-mapping in this region revealed a maximum two-point lod score of 2.8 at rs 2023865 and a maximum multipoint lod score of 17.9 at rs9304878. By haplotype construction, a (conservatively) approximately 17-cM critical region from SNP rs11084582 to SNP rs7258420 was defined. This region contains several neuronal genes that
\end{abstract}

constitute tantalizing etiological candidates for ETINPH. Conclusion: The importance of uncovering the genetic etiology of this disorder is irrefutable, as such a discovery could offer valuable insights into ET, iNPH, and related neurological conditions.

Copyright $\odot 2010$ S. Karger AG, Basel

\section{Introduction}

With the prevalence of essential tremor (ET) as high as $5-6 \%$ of the population $[1,2]$, ET represents the most common movement disorder interfering with activities of daily living $[1,3]$. In some studies it is as much as 20 fold more prevalent than Parkinson's disease [2-11]. Epidemiological research provides evidence that $\mathrm{ET}$ is often familial [7, 10, 12-15]. This finding likely led to initial descriptions of ET as 'familial tremor' [8, 9, 16, 17].

Idiopathic normal pressure hydrocephalus (iNPH) has its onset in geriatric years and is characterized by gait impairment, incontinence, and dementia. Anatomically, iNPH patients feature ventricular enlargement in the absence of elevated intracranial pressure. While about $40 \%$ of the congenital hydrocephalus cases are believed to have a possible genetic basis, no generally accepted genetic etiology for iNPH has been reported in the literature [18]. Tremor has occasionally been reported as a

\section{KARGER}

Fax +4161306 1234 E-Mail karger@karger.ch www.karger.com

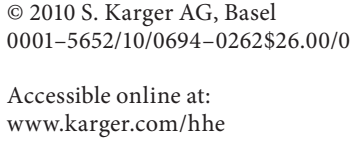

Jun Zhang, $\mathrm{ScD}, \mathrm{PhD}$

Departments of Anesthesiology and Biomedical Sciences, Center for Neurosciences

Texas Tech University Health Science Center

4800 Alberta Avenue, El Paso, TX 79905 (USA)

Tel. +1 915545 6560, Fax +1 915545 6984, E-Mail jun.zhang2000@gmail.com 
Fig. 1. The pedigree of a five-generation ETINPH family. Blood samples have been collected from the numbered individuals. Circles represent females; squares represent males. Black symbols are affected individuals, white symbols are normal individuals, and gray symbols are individuals with unknown status. For the genome scans, the two individuals in parentheses (II-2, II-3) comprise the founding members of each split pedigree branch. Underlined individuals are persons added to the opposite pedigree for the subsequent overlapped split linkage analysis.

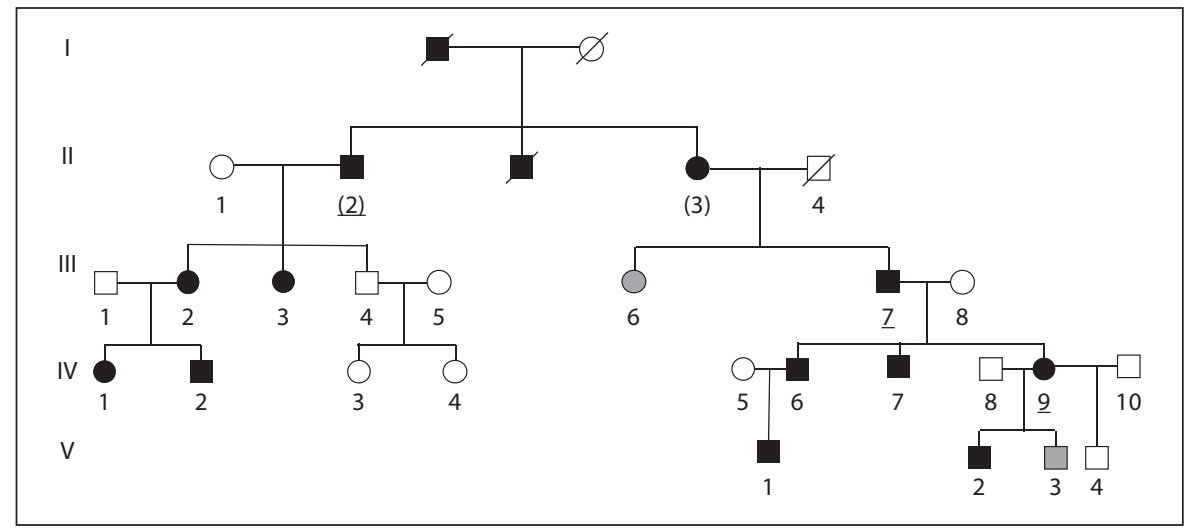

symptom of iNPH but is usually considered to be a Parkinsonian-type tremor [19-22].

In a previous report [23], we identified a five-generation kindred that bridged the gap between ET and iNPH, as multiple members of this family presented with ET in the adolescent or young adult years and three with additional iNPH when elderly. This disorder was named essential tremor-idiopathic normal pressure hydrocephalus (ETINPH). We hypothesized that the same neuronal gene was responsible for both the ET and the iNPH aspects of ETINPH. However, at the time, the identity and location of this putative gene were unknown. Using several different methods of linkage analysis, we have now identified the location of the gene down to a segment of chromosome 19. In this critical region, we have also identified several neuronal genes that constitute tantalizing candidates for this putative gene.

\section{Subjects and Methods}

\section{Subjects}

All research subjects are related to each other within a large kindred in which ET segregated as an autosomal dominant trait within the span of five generations. While the onset age of ET clusters in the early teens, the onset of iNPH occurs in affected subjects older than 65 years ( 3 affected subjects in the second generation). The recruiting process, clinical diagnostic criteria, and sample collection have been reported previously [23].

\section{DNA Genotyping}

To ensure robust results, two different single nucleotide polymorphism (SNP) genotyping microarrays were used: the Nsp array containing 262,000 SNPs from the GeneChip ${ }^{\circledR}$ Human Mapping 500K Array Set (Affymetrix) and the LINKAGE-12 panel containing 6,090 SNPs (Illumina). The genetic localization and allele frequencies of each SNP and the relevant genetic maps for candidate loci came from publicly available databases or were pro- vided by the manufacturers (Affymetrix and Illumina). Both arrays share some identical SNPs but with a relatively low concordance rate, which provides a great QC for both genotyping and platform. All SNP microarrays were genotyped at Yale Keck genomic core facility using standard protocols provided by the manufacturers. After the raw genotyping data had been generated from different platforms, the highest quality SNPs were further selected based on criteria such as minor allele frequency of $1 \%$, internal control markers, Mendelian consistency, and genotyping call rate of at least $93 \%$ as manufacture recommended, using $\mathrm{Ge}$ notyping Console and Bead Studio programs for Affymetrix and Illumina platforms, respectively.

\section{Linkage Analysis}

Prior to linkage analysis, the linkage utility softwares Pedcheck and Mega2 were used to check the genotyping data and transform the linkage file formats from different linkage analysis programs, respectively $[24,25]$. During the genome-wide scan, a model-dependent and a nonparametric linkage analysis of dense SNPs arrays were performed using sparse gene flow tree algorithms as implemented in Merlin [26], with a splitting approach due to the large size of the pedigree [27]. For fine-mapping, with the parameters of an autosomal dominant trait with a rare disease frequency of 0.0001 and full penetrance [23], a two-point modeldependent linkage analysis of the full pedigree was performed using the genotype/phase elimination algorithms implemented in LODLINK in the S.A.G.E. package [28], and a multipoint linkage analysis was performed using Markov chain-Monte Carlo approaches implemented in $1 \mathrm{~m} \_$lods in the Morgan package [29].

\section{Results}

After carefully reviewing the medical condition of each participant based on our clinical criteria, we determined that further medical evaluation was needed for two individuals previously diagnosed as 'affected', and redefined their status as 'unknown' before this analysis to ensure the conservativeness of linkage analysis (fig. 1), re- 

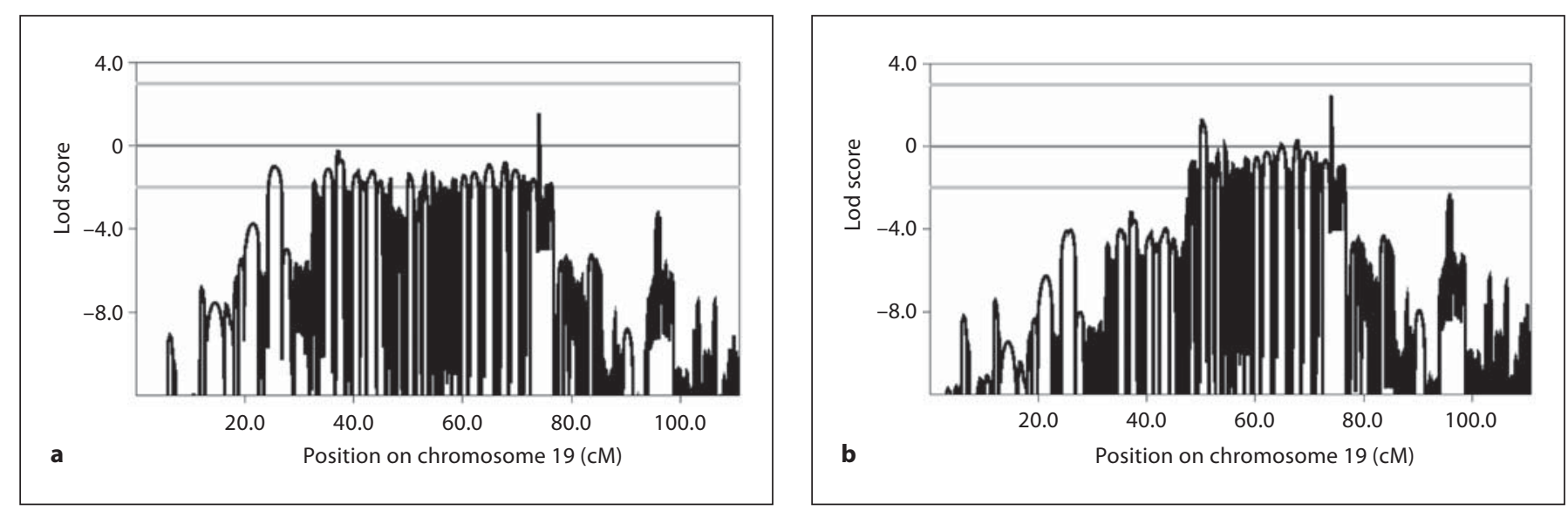

Fig. 2. During genome scan using Merlin, the peak of lod scores on chromosome 19 increases from the split pedigree analysis (a) to reach almost 3.0 in the overlapped split pedigree analysis (b) in Affychips, indicating a potential candidate site for the ETINPH locus.

sulting in subtle changes compared to our previously described pedigree [23]. We performed a genome-wide linkage scan with two split pedigrees (the II- 2 branch and II-3 branch, fig. 1) first, using the linkage program Merlin. Merlin is the best program to handle linkage analysis with high-density SNPs, but it can only handle around 14 individuals at its maximal capacity. In both model-dependent and nonparametric analyses with Merlin, five peaks on chromosomes 10, 16, 17 and 19 with significantly large lod scores were found using Affymetrix chips (Affychips); only two peaks on chromosome 10 and 19 with large lod scores were found using Illumina chips. While the locations of the peaks on chromosome 10 found in the Affymetrix and Illumina chips did not overlap, the locations of the peaks on chromosome $19 \mathrm{did}$.

Next, we performed another round of genome-wide linkage scan with two overlapped split pedigrees (the II-2 pedigree with two more affecteds from the II-3 branch, and the II-3 pedigree with one more affected from the II-2 branch). In Affychips, only two peaks (chromosome 16 and 19) remained with increased lod scores in both model-dependent and nonparametric analysis. In Illumina chips, both peaks on chromosome 10 and 19 defined in the previous analysis remained and had increased lod scores in model-dependent and nonparametric analysis. The peak on chromosome 19 still represented the only common region in this round of analysis, with a maximum model-dependent lod score of 2.4 (fig. 2). Therefore, we concluded that the genetic locus of ETINPH is in this segment of chromosome 19.
Fine-mapping using LODLINK and two-point modeldependent linkage analysis for the full pedigree revealed significant lod scores among SNPs in this interval (more than 20 SNPs with maximum two-point lod scores around 2.0), with their recombination fractions $(\theta)$ at zero. A maximum two-point lod score of 2.8 was found at rs2023865. This finding in combination with haplotype construction places the ETINPH gene in an approximately $17-\mathrm{cM}$ critical interval from rs11084582 to rs7258420 on chromosome 19q12-13.31 (fig. 3). Among 407 SNPs from the Nsp array in the 17-cM interval (approximately $13.2 \mathrm{Mb}$ ), only 29 SNPs with an average genetic distance of $0.605 \mathrm{cM}$ were chosen in order to perform the multiple linkage analysis in an attempt to limit the potential inflation in the multipoint lod score due to the possibility of linkage disequilibrium. In multipoint linkage analysis using Markov chain-Monte Carlo approaches, 9 out of 10 runs in lm_lods generated positive results with a lod score $>3.0$. Two maximum multipoint lod scores reached 17.9 (rs9304878), further corroborating the linkage of the ETINPH gene to this interval (fig. 3).

\section{Discussion}

Through this genome-wide linkage screening, we have mapped the ETINPH locus to chromosome 19q12-13.31. The current interval is relatively large (approximately 17 $\mathrm{cM}$ ) based on our conservative approach. In a next stage, more powerful next-generation genomic sequencing 
Fig. 3. Graph of chromosome 19 coordinates versus two-point lod scores generated from LODLINK (dashed line) and the multipoint lod scores maximized from lm_lods (solid line).

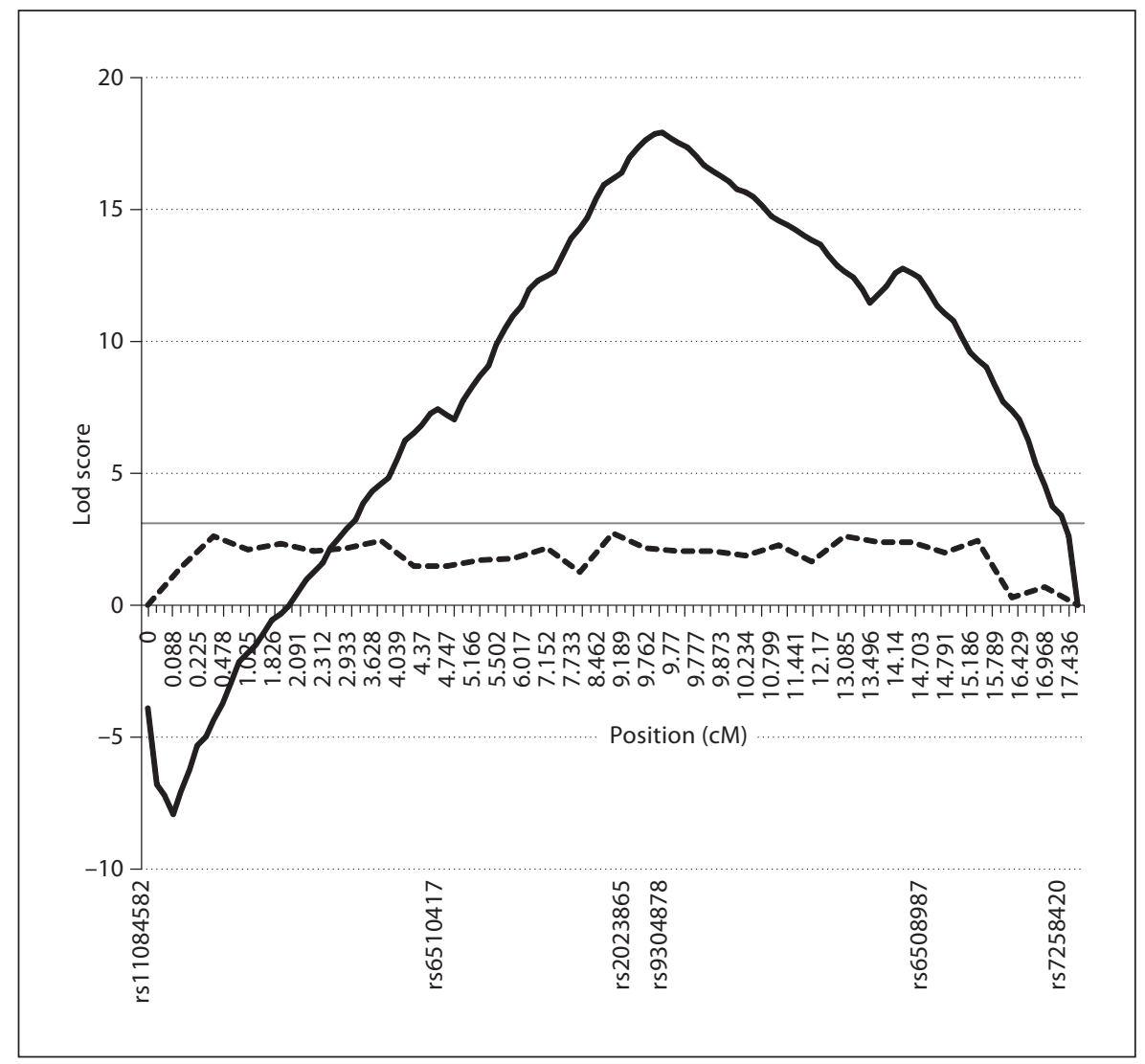

techniques will be applied to sequence the entire interval which will lead to rapid cloning of the ETINPH gene. Within the current large interval, we have selected several potential candidate genes whose products are required for the proper functioning of the nervous system. As such, mutations in these genes represent possible explanations for the pathology seen in ETINPH.

The first of these genes is ATP1A3. This gene codes for an isoform of the alpha subunit of N,K-ATPase that is expressed in the nervous system. A mutation in ATP$1 \mathrm{~A} 3$ has been found to cause the autosomal dominant disorder called rapid-onset dystonia-parkinsonism [30]. In this disease, patients between the ages of 14 and 45 years develop involuntary spasms of the extremities, bradykinesia, dysarthria, dysphagia, and postural instability [31-33]. Several features of this disease resemble ETINPH. Most obviously, both feature a central movement disorder. Additionally, the age at onset of rapidonset dystonia-parkinsonism parallels that of ET in ETINPH, and both disorders are inherited in an autosomal dominant manner. These intriguing similarities suggest a possible link between ET and parkinsonism, as has been suggested previously [34]. There is a possibility that different mutations of ATP1A3 that produce varying degrees of function loss may lead to different clinical manifestations.

Another noteworthy finding within the chromosomal segment identified by linkage is a collection of genes whose malfunction may perturb the biochemical pathway that has been implicated in Alzheimer's disease. Among these genes is presenilin enhancer 2 (PSENEN). The protein product of PSENEN enhances the transcription of presenilin, a component of gamma-secretase. Gamma-secretase is involved in the cleavage of amyloid precursor protein (APP); inappropriate cleavage of APP can cause Alzheimer's disease. Indeed, prior research has shown that mutations in presenilins and APP can cause familial forms of Alzheimer [35]. In addition to PSENEN, amyloid beta A4 precursor-like protein 1 (APLP1) resides within the identified segment of chromosome 19. APLP1 is structurally homologous to APP [36]; this structural similarity may imply that a mutation of APLP1 could engender Alzheimer-like pathology. Disruption of either the same or a similar biochemical pathway as Alzhei- 
mer's disease could possibly account for the features of ETINPH that resemble Alzheimer's disease, including dementia and enlarged ventricles.

In addition to the aforementioned candidate genes, other genes that directly impact the nervous system reside in the chromosomal segment 19q12-13.31. CAPN12, a cysteine protease, contributes to synaptic plasticity [37]. LGI4 is highly expressed in the brain, and mutations of genes in the same family have been associated with epilepsy [38]. MAG encodes for a protein in myelin that inhibits neurite outgrowth and plasticity [39]. DPF1 is the gene for a neural-specific zinc finger protein [40]. CIC is a repressor of transcription that may influence CNS development; high CIC expression has been found in CNS tumors [41]. ETHE1 and PRX are both located within 19q12-13.31, but the neurological sequelae of mutations in these genes (ethylmalonic encephalopathy and Deferine-Sottas syndrome, respectively) are already well documented $[42,43]$.

In summary, although we have selected several candidate genes in the interval to discuss, it has to be empha- sized here that the phenotypic spectum of ETINPH does mostly not overlap with any of the clinical manifestations caused by those genes. It is our hope that identification of the ETINPH gene will help us to understand the molecular mechanism of how the shared pathophysiological pathway contributes to both diseases. A better understanding of this common pathophysiological pathway will further aid in our understanding of other related neurological conditions. Our current molecular cloning effort will definitely give us a clear answer as to the genetic etiology of ETINPH.

\section{Acknowledgements}

The authors are grateful to the patient family for their enthusiastic support in the study. We also wish to thank Michael Williams at the Hydrocephalus Research Center in LifeBridge Health Brain \& Spine Institute for his kind help during patient recruitment and evaluation and Jared Marks at UMMC Neurosurgery for his technical support in the laboratory.

\section{References}

$\checkmark 1$ Louis ED, Barnes L, Albert SM, et al: Correlates of functional disability in essential tremor. Mov Disord 2001;16:914-920.

-2 Murray TJ: Essential tremor. Can Med Assoc J 1981;124:1559-1565, 1570.

-3 Benito-Leon J, Louis ED: Essential tremor: emerging views of a common disorder. Nat Clin Pract Neurol 2006;2:666-678; quiz 691-693.

4 Larsson T, Sjogren T: Essential tremor: a clinical and genetic population study. Acta Psychiatr Scand 1960;36(suppl 144):1-176.

5 Ashenhurst EM: The nature of essential tremor. Can Med Assoc J 1973;109:876-878.

-6 Larsen TA, Calne DB: Essential tremor. Clin Neuropharmacol 1983;6:185-206.

7 Hubble JP, Busenbark KL, Koller WC: Essential tremor. Clin Neuropharmacol 1989;12: 453-482.

-8 Metzer WS: Essential tremor: an overview. J Ark Med Soc 1994;90:587-590.

$\checkmark 9$ Louis ED: Essential tremor. Arch Neurol 2000;57:1522-1524.

10 Jankovic J, Madisetty J, Vuong KD: Essential tremor among children. Pediatrics 2004;114: 1203-1205.

-11 Louis ED: Essential tremor. Lancet Neurol 2005;4:100-110.

12 Sutherland JM, Edwards VE, Eadie MJ: Essential (hereditary or senile) tremor. Med J Aust 1975;2:44-47.
3 Louis ED, Ottman R: How familial is familial tremor? The genetic epidemiology of essential tremor. Neurology 1996;46:12001205.

14 Louis ED, Ottman R, Ford B, et al: The Washington Heights-Inwood Genetic Study of Essential Tremor: methodologic issues in essential-tremor research. Neuroepidemiology 1997;16:124-133.

15 Louis ED, Barnes LF, Ford B, Ottman R: Family history information on essential tremor: potential biases related to the source of the cases. Mov Disord 2001;16:320-324.

$\checkmark 16$ Schade H: Hereditary problems in a family with essential hereditary tremor (in German). Z Mensch Vererb Konstitutionsl 1956; 33:355-364.

17 Lorenz D, Frederiksen H, Moises H, Kopper F, Deuschl G, Christensen K: High concordance for essential tremor in monozygotic twins of old age. Neurology 2004;62:208211.

18 Zhang J, Williams MJ, Rigamonti D: Genetics of human hydrocephalus. J Neurology 2006;253:1255-1266.

19 Lieberman AN: Parkinson's disease: a clinical review. Am J Med Sci 1974;267:66-80.

20 Kandel EI, Mirkiev AM: [Parkinsonism and hydrocephalus]. Zh Nevropatol Psikhiatr Im S S Korsakova 1979;79:917-922.
1 Gabellini AS, Martinelli P, Gulli MR, Ambrosetto G, Ciucci G, Lugaresi E: Orthostatic tremor: essential and symptomatic cases. Acta Neurol Scand 1990;81:113-117.

22 Relkin N, Marmarou A, Klinge P, Bergsneider M, Black PM: Diagnosing idiopathic normal-pressure hydrocephalus. Neurosurgery 2005;57:S4-S16; discussion ii-v.

23 Zhang J, Williams MA, Rigamonti D: Heritable essential tremor-idiopathic normal pressure hydrocephalus (ETINPH). Am J Med Genet A 2008;146A:433-439.

24 O'Connell JR, Weeks DE: PedCheck: a program for identification of genotype incompatibilities in linkage analysis. Am J Hum Genet 1998;63:259-266.

25 Mukhopadhyay N, Almasy L, Schroeder M, Mulvihill WP, Weeks DE: Mega2: data-handling for facilitating genetic linkage and association analyses. Bioinformatics 2005;21: 2556-2557.

26 Abecasis GR, Cherny SS, Cookson WO, Cardon LR: Merlin - rapid analysis of dense genetic maps using sparse gene flow trees. Nat Genet 2002;30:97-101.

27 Bellenguez C, Ober C, Bourgain C: A multiple splitting approach to linkage analysis in large pedigrees identifies a linkage to asthma on chromosome 12. Genet Epidemiol 2009; $33: 207-216$ 
28 S.A.G.E: Statistical Analysis for Genetic Epidemiology. Release 600, 2009; http://darwin. cwru.edu/.

-29 Wijsman EM, Rothstein JH, Thompson EA: Multipoint linkage analysis with many multiallelic or dense diallelic markers: Markov chain-Monte Carlo provides practical approaches for genome scans on general pedigrees. Am J Hum Genet 2006;79:846-858.

30 de Carvalho Aguiar P, Sweadner KJ, Penniston JT, et al: Mutations in the $\mathrm{Na}^{+} / \mathrm{K}^{+}$-ATPase alpha3 gene ATP1A3 are associated with rapid-onset dystonia parkinsonism. Neuron 2004;43:169-175.

-31 Brashear A, DeLeon D, Bressman SB, Thyagarajan D, Farlow MR, Dobyns WB: Rapidonset dystonia-parkinsonism in a second family. Neurology 1997;48:1066-1069.

\32 Dobyns WB, Ozelius LJ, Kramer PL, et al: Rapid-onset dystonia-parkinsonism. Neurology 1993;43:2596-2602.
33 Zaremba J, Mierzewska H, Lysiak Z, Kramer P, Ozelius LJ, Brashear A: Rapid-onset dystonia-parkinsonism: a fourth family consistent with linkage to chromosome 19q13. Mov Disord 2004;19:1506-1510.

34 Shahed J, Jankovic J: Exploring the relationship between essential tremor and Parkinson's disease. Parkinsonism Relat Disord 2007;13:67-76.

35 Hutton M, Hardy J: The presenilins and Alzheimer's disease. Hum Mol Genet 1997;6: 1639-1646.

36 Wasco W, Brook JD, Tanzi RE: The amyloid precursor-like protein (APLP) gene maps to the long arm of human chromosome 19. Genomics 1993; 15:237-239.

-37 Dear TN, Meier NT, Hunn M, Boehm T: Gene structure, chromosomal localization, and expression pattern of Capn12, a new member of the calpain large subunit gene family. Genomics 2000;68:152-160.

$38 \mathrm{Gu}$ W, Wevers A, Schroder H, et al: The LGI1 gene involved in lateral temporal lobe epilepsy belongs to a new subfamily of leucinerich repeat proteins. FEBS Lett 2002;519:7176
39 Domeniconi M, Cao Z, Spencer T, et al: Myelin-associated glycoprotein interacts with the Nogo66 receptor to inhibit neurite outgrowth. Neuron 2002;35:283-290.

$\checkmark 40$ Chestkov AV, Baka ID, Kost MV, Georgiev GP, Buchman VL: The d4 gene family in the human genome. Genomics 1996;36:174-177.

41 Lee CJ, Chan WI, Scotting PJ: CIC, a gene involved in cerebellar development and ErbB signaling, is significantly expressed in medulloblastomas. J Neurooncol 2005;73:101108.

42 Tiranti V, D’Adamo P, Briem E, et al: Ethylmalonic encephalopathy is caused by mutations in ETHE1, a gene encoding a mitochondrial matrix protein. Am J Hum Genet 2004;74:239-252.

43 Boerkoel CF, Takashima H, Stankiewicz P, et al: Periaxin mutations cause recessive Dejerine-Sottas neuropathy. Am J Hum Genet 2001;68:325-333. 